\title{
Análisis estadístico de la educación matemática en la ciudad de Pereira
}

\author{
Statistical analysis of mathematics education in the city of Pereira.
}

\author{
Adriana María Yepes Montoya, José Rubiel Bedoya Sánchez \\ Facultad de Ciencias Básicas, Universidad Tecnológica de Pereira, Colombia \\ $\frac{\text { adril } 742 \text { ahotmail.com }}{\text { joserubieldutp.edu.co }}$
}

\begin{abstract}
Resumen- A partir de esta investigación se pretende determinar la situación actual de la educación matemática en la ciudad de Pereira, consultando sobre el nivel de conocimientos matemáticos, los errores conceptuales y la actitud que hacia la matemática escolar presentan tanto estudiantes como profesores de las I. E. oficiales de la ciudad de Pereira. Todos estos aspectos se detectaron basados en instrumentos de medición acordes para cada caso y construidos de acuerdo a los aspectos teóricos señalados por el Ministerio de Educación Nacional en sus estándares curriculares de matemáticas y por el Instituto para el Fomento a la Educación Superior ICFES para pruebas Saber, de igual forma se tuvo en cuenta las teorías sobre actitudes y se usó como escala de medición la escala tipo Likert, los resultados encontrados fueron bajos tanto en estudiantes como en docentes, panorama que se puede corroborar con los actuales resultados de las pruebas nacionales e internacionales. Estos resultados son producto de factores multicausales.
\end{abstract}

Palabras clave - Análisis descriptivo, análisis inferencial, actitud matemática, conocimientos matemáticos.

Abstract- From this investigation is to determine the current status of mathematics education in the city of Pereira, at about the level of mathematical knowledge, misconceptions and attitude towards school mathematics presented both students and teachers of the official IE the city of Pereira. All these aspects by the Institute for Higher Education Development test ICFES Saber were detected based on consistent measurement tools for each case and built according to the theoretical issues raised by the Ministry of Education in their curriculum standards for mathematics and, likewise took into account the attitudes and theories used as measurement scale Likert scale, the results found were low in both students and teachers, scenario that can be corroborated with the present results of national and international events. These results are the product of multi-causal factors.

Key Word - Descriptive analysis, inferential analysis, mathematics attitude, mathematical knowledge.

\section{INTRODUCCIÓN}

El panorama internacional, nacional y local en materia de educación no es alentador, en los últimos años las pruebas internacionales han dejado a Colombia ubicada en los últimos lugares y a nivel nacional y local los estudiantes sólo alcanzan un nivel medio de desempeño, situación preocupante por la competitividad y la era de globalización en que nos encontramos.

Debido a las dificultades tanto en la enseñanza, como en el aprendizaje de las matemáticas en todos los niveles escolares, la Educación Matemática ha causado en las últimas décadas, especial interés en académicos e investigadores a nivel internacional, nacional y regional. Por esta razón se hace muy importante identificar los niveles de aprendizaje con que cuentan los estudiantes en el área de matemáticas en los grados $3^{\circ}, 5^{\circ}, 9^{\circ}$, y $11^{\circ}$ (grados en los que se aplican las pruebas Saber), así como los factores influyentes en el bajo rendimiento académico y como estos están asociados con la actitud que tanto estudiantes como docentes presentan hacia ella, es por esto que con esta investigación se pretende responder a las preguntas: ¿cuál es la situación actual de la enseñanza y el aprendizaje de las matemáticas en el nivel de básica y media en la ciudad de Pereira?, ¿qué nivel de conocimientos poseen los estudiantes y profesores?, ¿cuáles son los algunos de los errores conceptuales más comunes que se presentan en estudiantes y docentes? y ¿cuál es la actitud que poseen frente a la matemática? aspectos de gran interés para toda la comunidad educativa.

\section{CONTENIDO}

\section{A. Fundamentos del estudio}

Como es sabido el nivel educativo en el área de matemáticas es medido por diferentes entidades nacionales e internacionales. Un ejemplo son la pruebas internacionales TIMSS que evalúan el nivel en matemáticas y ciencias en 
estudiantes de grado cuarto de primaria y grado octavo de secundaria, para esta prueba los resultados de 2007 (1), establecen como puntaje promedio internacional un valor de 500 puntos en ambos grados, según esta referencia, Colombia con un puntaje promedio para grado cuarto de 355 puntos se encuentra ubicado en la posición 30 de 36 participantes (Hong Kong el país mejor ubicado sacó 607 puntos), y en grado octavo un puntaje promedio de 380 puntos ubica al país en la posición 39 de 47 participantes (mientras Taipéi el país mejor ubicado obtuvo 598 puntos), además ningún estudiante colombiano alcanzó los niveles avanzados de desempeño en la prueba y más del $60 \%$ de los estudiantes no alcanzó el nivel mínimo establecido, cabe decir que los resultados de estas pruebas son similares a los obtenidos en la evaluación PISA, (2), en el año 2012 el puntaje de Colombia (376) es inferior a los obtenidos por 61 países y no es estadísticamente diferente de los observados en los países que obtuvieron los tres puntajes más bajos: Catar, Indonesia y Perú. Siendo matemáticas la prueba con peores resultados de las tres áreas evaluadas (matemáticas, lectura y ciencias).

En cuanto a niveles de competencia en matemáticas, el $74 \%$. de los estudiantes colombianos se ubicó por debajo del nivel 2 que según PISA es el nivel de competencia insuficiente, el $18 \%$ se ubicó en el nivel 2 (básico) y sólo 3 de cada mil estudiantes alcanzaron los niveles 5 y 6 (niveles de pensamiento y razonamiento matemático avanzado).

En cuanto a Pereira aproximadamente el $0,2 \%$ se encuentra en los niveles 5 y 6 , el $16,7 \%$ en el nivel 2 y el $75,5 \%$ por debajo del nivel básico.

Este panorama internacional muestra las deficiencias que el país tiene en el desempeño escolar en el área de matemáticas y como urge la necesidad de mejorar la educación matemática en él. Así mismo el ICFES (3) realiza esta medición, a través de las pruebas saber para grado tercero, quinto, noveno y once, permitiendo conocer el nivel nacional en el área de matemáticas y ubicar departamentos y municipios respecto a los resultados en ella. Los resultados nacionales de las pruebas saber 2009, y 2012 (4) muestran los promedios generales en la prueba de matemáticas y en todos ellos se observa que los estudiantes colombianos tienen un nivel mínimo de desempeño en los grados tercero, quinto y noveno, y un nivel medio para grado once, según los niveles establecidos por el MEN.

Los resultados también detectan que los niveles de desempeño en las instituciones oficiales urbanas en la ciudad de Pereira en cuanto a matemáticas presentan un alto porcentaje de estudiantes en el nivel insuficiente (4). En el año 2012 en el grado tercero el $18 \%$ se encontraba en la categoría de desempeño insuficiente, en grado quinto el $29 \%$ y en grado noveno el $15 \%$ se encontraban en la misma categoría.

En la categoría avanzado en el año 2012, en el grado tercero se encontraba el $15 \%$, grado quinto el $11 \%$ y en grado noveno sólo un $3 \%$ se encontraba en esta misma categoría.

\section{B. Metodología de la investigación}

El desarrollo de este proyecto se llevó a cabo a través de una metodología cuantitativa, en donde la población objeto de estudio son los estudiantes y profesores de educación básica y media de las instituciones oficiales de la ciudad de Pereira en la zona urbana durante el año 2013, la muestra se realizó en 17 instituciones para un total de 230 estudiantes y 44 profesores, realizando sobre ella un muestreo aleatorio estratificado (por nivel escolar: $3^{\circ}, 5^{\circ}, 9^{\circ}$ y $11^{\circ}$ ) en varias etapas:

Primera etapa: Se hizo una selección de instituciones, con probabilidad proporcional al tamaño por grado, (el tamaño se definió como la cantidad de estudiantes).

Segunda Etapa: Selección de grupos con muestreo aleatorio simple (M.A.S).

Tercera Etapa: Selección de estudiantes con muestreo aleatorio simple (M.A.S).

Los conocimientos y errores conceptuales se midieron en profesores de primaria y secundaria y en estudiantes de los grados $3^{\circ}, 5^{\circ}, 9^{\circ}$ y $11^{\circ}$, buscando con esto analizar los procesos educativos al final de cada nivel escolar: básica primaria, básica secundaria y media.

La prueba de conocimiento matemático se elaboró con preguntas tipo ICFES y constaba de 25 preguntas, 5 por cada pensamiento matemático y además de medir el nivel de conocimiento matemático tenían un componente adicional, que consistía en que las opciones múltiples de respuesta para cada pregunta, tenían una intencionalidad de respuesta que permitía determinar los errores conceptuales en cada caso.

En el caso de los profesores se les aplicó la misma prueba de conocimientos de grado quinto para los profesores de primaria, y la prueba de grado once para los profesores de matemáticas de básica y media. Esto se hizo teniendo en cuenta los conocimientos mínimos que deben poseer los docentes en cada nivel y la valoración se hizo de la misma forma que para los estudiantes.

$$
\begin{array}{llll}
\text { III. RESULTADOS } & \text { DE } & \text { LA } \\
\text { INVESTIGACIÓN } & &
\end{array}
$$

El análisis de frecuencias de respuestas a las pruebas de conocimientos, permitió detectar los errores conceptuales más comunes en el área de matemáticas, tanto de estudiantes como de profesores, encontrando que existen falencias en temas fundamentales y básicos en cada pensamiento matemático y que estos errores persisten al pasar de un nivel a otro. Algunos de esos errores conceptuales son:

En pensamiento numérico en estudiantes: 
En grado tercero más del $50 \%$ de los estudiantes presentan dificultades para identificar el valor posicional de un número, reconocer múltiplos y divisores, realizar secuencias numéricas y establecer relaciones de orden. En grado quinto más del $70 \%$ tienen dificultades con el concepto de cantidad, y con operaciones básicas de números enteros, fraccionarios y decimales. En grado noveno en más del $50 \%$ hay falencias en operaciones básicas con números reales, con las operaciones de potenciación, radicación y logaritmación, con planteamiento y resolución de ecuaciones y determinantes y en grado once más del $60 \%$ tienen dificultades al realizar operaciones entre conjuntos, plantear y resolver ecuaciones, y con el concepto y representación de funciones.

En el caso de los profesores, el 32\% de los profesores de básica primaria obtuvo puntajes inferiores al $50 \%$ en este pensamiento; en secundaria el $75 \%$ presento falencias en operaciones con conjuntos, el $56 \%$ en identidades trigonométrica y el $62,5 \%$ obtuvo puntajes inferiores al $50 \%$.

En pensamiento variacional en los estudiantes:

En grado tercero se encontraron dificultades en más del $60 \%$ de los estudiantes al identificar los términos de una serie o secuencia numérica, en grado quinto en más del $70 \%$ al identificar posiciones decimales, representación de un numero mixto, representación de una potencia, variación de un costo y proporciones, en grado noveno en más del $60 \%$ se detectan falencias al aplicar propiedades de potenciación, resolver sistemas de ecuaciones lineales y al plantear y representar una función lineal y en grado once en más del $77 \%$ hay falencias al plantear y resolver ecuaciones, reconocer funciones en diferentes representaciones y con los conceptos de dominio y rango.

En el caso de los profesores de primaria, el 68\% presenta dificultades con proporciones entre magnitudes, el 28,5\% confunde las posiciones decimales de un número, el $25 \%$ obtuvo puntajes inferiores al $60 \%$.

El $25 \%$ de los docentes de secundaria obtuvo puntajes inferiores al $80 \%$.

En pensamiento métrico en estudiantes:

En grado tercero más del $47 \%$ tienen dificultades al medir una longitud y no reconocen las unidades de medida, no conocen la medición del tiempo en el reloj, ni del peso en la báscula y el $94 \%$ no conoce el concepto de área. En grado quinto el $88 \%$ confunden los conceptos de área y volumen y no conocen como hallarlos, ni hacen conversión de unidades. En grado noveno más del $80 \%$ no reconocen ni hallan áreas ni volúmenes, ni identifican la proporcionalidad de lados de un triángulo. En grado once más del $70 \%$ no identifican ni aplican el teorema de Pitágoras, ni el de seno ni el de coseno, ni reconocen ni hallan perímetro, áreas o volúmenes ni realizan conversión de unidades, no conocen el proceso para hallar la distancia entre dos puntos.
En el caso de los profesores de primaria, El ítem que indagaba sobre el concepto de área y conversión de unidades, sólo lo respondió correctamente 1 docente $(3,6 \%)$, y no la respondió el $35,7 \%$. El $57,1 \%$ obtuvo puntajes inferiores al $60 \%$.

En los profesores de secundaria, el porcentaje de no respuesta para algunos ítems llegó hasta el 43,8\%. El 56,3\% de los profesores obtuvo puntajes inferiores al $60 \%$ en este componente.

En pensamiento geométrico en estudiantes:

En grado tercero más del $50 \%$ no conocen las figuras geométricas básicas, no realizan transposición de figuras, en grado quinto más del $86 \%$ no diferencia los triángulos ni sus elementos, no identifica el desarrollo plano de un cuerpo geométrico, ni identifica ángulos congruentes. En grado noveno el $60 \%$ no tiene claro el concepto de polígono y más del $76 \%$ no conoce los criterios de semejanza de triángulos, ni interpreta el teorema de Pitágoras ni tiene claro el concepto de volumen ni área. En grado once más del $62 \%$ no identifican las funciones trigonométricas, ni conocen el concepto de poliedro, ni la ecuación de una elipse, ni el concepto de discontinuidad.

En los profesores de primaria, El 28,6\% contestó erróneamente, mostrando falencias en el concepto de ángulos agudos, en todos los ítems el porcentaje de no respuesta fue del $25 \%$ y el $39,3 \%$ presentan puntajes inferiores al $60 \%$.

En los profesores de secundaria, el 56,4\% presenta dificultades al encontrar la razón entre volúmenes de diferentes cuerpos geométricos, el porcentaje de no respuesta en cada ítem estuvo entre el $37,5 \%$ y el $43,8 \%$ y el $93,8 \%$ obtuvo puntajes inferiores al $60 \%$ en este componente.

En pensamiento aleatorio en estudiantes:

En grado tercero el $67 \%$ no tiene claro el concepto de combinación. En grado quinto el $90 \%$ no interpreta datos estadísticos en diferentes representaciones, el72\% no interpreta la combinación, el $64 \%$ confunde las medidas de tendencia central y el $86 \%$ no tiene claro el concepto de probabilidad. En grado noveno el $60 \%$ no interpreta tablas ni gráficos, el $90 \%$ confunde las medidas de tendencia central y no reconocen un dato atípico, el $86 \%$ no hallan probabilidades y el $64 \%$ no elaboran diagramas de árbol. En grado once el $97 \%$ confunden los conceptos de espacio muestral y tamaño del evento y no hallan probabilidades, el $78 \%$ no conocen las técnicas de conteo y el $50 \%$ no interpretan diagramas circulares.

En los profesores de primaria, El 71,4\% presenta dificultades con la interpretación de datos desde diferentes representaciones, el 46,4\% con la relación entre un grafico de barras y su respectiva tabla de valores, el 53,6\% obtuvo puntajes inferiores al $60 \%$ en este componente. 
Al analizar el desempeño general por grados se encontró según la prueba Chi-cuadrado que existe asociación entre las variables desempeño general y grado, y de acuerdo al coeficiente de correlación de Spearman esta asociación es inversa, es decir, a medida que aumenta el grado de los estudiantes disminuye el nivel de desempeño escolar y como muestra la tabla 1, el nivel de desempeño general es bajo, según la clasificación dada por el ICFES.

\begin{tabular}{|c|c|c|c|c|c|c|}
\hline \multicolumn{2}{|c|}{} & \multicolumn{3}{|c|}{ Desempeño general } & \multicolumn{2}{c|}{$\begin{array}{c}\text { Puntaje } \\
\text { Porcentual }\end{array}$} \\
\cline { 2 - 7 } \multicolumn{2}{|c|}{} & Bajo & $\begin{array}{c}\text { Medio } \\
\text { bajo }\end{array}$ & Medio & Media & IC \\
\hline \multirow{4}{*}{ Grado } & $3^{\circ}$ & $15,70 \%$ & $41,40 \%$ & $42,90 \%$ & 45,68 & $\begin{array}{c}37,80- \\
53,55\end{array}$ \\
\cline { 2 - 7 } & $5^{\circ}$ & $58,00 \%$ & $38,00 \%$ & $4,00 \%$ & 27,68 & $\begin{array}{c}15,54- \\
39,82\end{array}$ \\
\cline { 2 - 7 } & $9^{\circ}$ & $54,00 \%$ & $42,00 \%$ & $4,00 \%$ & 30,88 & $\begin{array}{c}26,38- \\
35,38\end{array}$ \\
\cline { 2 - 7 } & $11^{\circ}$ & $60,00 \%$ & $35,00 \%$ & $5,00 \%$ & 28,73 & $\begin{array}{c}20,11- \\
37,36\end{array}$ \\
\hline $\begin{array}{c}\text { Total } \\
\text { Prueba }\end{array}$ & $44,80 \%$ & $39,10 \%$ & $16,10 \%$ & 33,24 & $\begin{array}{c}29,76- \\
36,71\end{array}$ \\
\hline
\end{tabular}

Tabla 1: Análisis por grado

En el análisis del puntaje porcentual por pensamiento matemático se encontró que el pensamiento numérico presenta el puntaje promedio más bajo de los 5 pensamientos correspondiente al nivel bajo, los demás pensamientos matemáticos se encuentran en el nivel medio bajo.

\begin{tabular}{|c|c|c|c|}
\hline Pensamiento & \multirow{2}{*}{ Media } & \multicolumn{2}{|c|}{ I. C al 95\% } \\
\hline Numérico & 28,43 & 23,99 & 32,86 \\
\hline Variacional & 32,89 & 27,62 & 38,16 \\
\hline Métrico & 33,67 & 28,52 & 38,82 \\
\hline Geométrico & 37,14 & 32,41 & 41,87 \\
\hline Aleatorio & 34,50 & 30,22 & 38,77 \\
\hline
\end{tabular}

Tabla 2: Análisis por pensamiento

Al puntaje porcentual general en conocimientos matemáticos por pensamiento y a la prueba de actitud hacia la matemática se le realizó análisis de varianza previa verificación de los supuestos de normalidad y homogeneidad de varianzas, en caso de no cumplirse los supuestos estadísticos se utilizó como alternativa la prueba no paramétrica de Krustall Wallis.

Dado que existen diferencias significativas en las medias se realizó la prueba T2 de Tamhane, ya que se probó que las varianzas no son iguales, encontrando que hay diferencias significativas entre las medias de grado $3^{\circ}$ con las medias de grado $5^{\circ}, 9^{\circ}$ y $11^{\circ}$ y en el pensamiento aleatorio se encontró diferencias significativas entre las medias de los grados $5^{\circ}$ y $9^{\circ}$.
Debido a que los datos difieren en sus promedios poblacionales, se procedió a realizar comparaciones y análisis con otras variables tenidas en cuenta en el estudio como la edad, el sexo y modalidad de la IE, entre otras.

Análisis de la prueba de conocimientos matemáticos y la prueba de actitud matemática frente a la edad del estudiante:

Según el coeficiente de correlación de Pearson ( $\rho=$ $-0,397$, con un $p-$ valor $<0,001$ ), existe relación inversa entre la edad y la prueba de conocimientos matemáticos y entre la edad y la prueba de actitud matemática, es decir, a mayor edad disminuye el puntaje en todos los pensamientos matemáticos y en la prueba general de conocimientos matemáticos y la actitud es más desfavorable, hecho que confirma los resultados anteriores.

Análisis de la prueba de conocimientos matemáticos y la escala de actitud matemática frente al sexo del estudiante:

La media de ambos sexos se encuentra en el nivel de desempeño medio bajo, con el $95 \%$ de los datos en este mismo nivel. La prueba de actitud hacia la matemática muestra que tanto los hombres como las mujeres presentan una actitud positiva hacia la matemática.

\begin{tabular}{|c|c|c|c|c|c|}
\hline \multirow{2}{*}{ Sexo } & \multicolumn{2}{|c|}{ Desempeño general } & \multicolumn{2}{c|}{$\begin{array}{c}\text { Puntaje } \\
\text { Porcentual }\end{array}$} \\
\cline { 2 - 6 } & Bajo & $\begin{array}{c}\text { Medio } \\
\text { bajo }\end{array}$ & Medio & Media & $\begin{array}{c}\text { I. C. al } \\
95 \%\end{array}$ \\
\hline Hombre & $38,80 \%$ & $43,40 \%$ & $17,80 \%$ & 35,96 & $\begin{array}{c}32,72- \\
39,19\end{array}$ \\
\hline Mujer & $48,20 \%$ & $36,70 \%$ & $15,10 \%$ & 32,95 & $\begin{array}{c}30,56- \\
35,34\end{array}$ \\
\hline
\end{tabular}

Tabla 3: Análisis por sexo

La diferencia de medias según el sexo se analizó a través la prueba $\mathrm{T}$ para muestras independientes, encontrando que no existen diferencias significativas entre las medias de los hombres y de las mujeres en ninguna de las dos pruebas.

$\mathrm{Al}$ analizar la variable desempeño general según el sexo de los estudiantes, según la prueba Chi-cuadrado de Pearson $(p=0,548)$ no existe asociación entre el nivel de desempeño y el sexo de los estudiantes.

Análisis de la prueba de conocimientos matemáticos y la prueba de actitud matemática frente a la modalidad de la institución:

El puntaje promedio de la prueba de conocimientos matemáticos muestra que la modalidad normalista es la que obtuvo el puntaje promedio más bajo. $\mathrm{Al}$ analizar el desempeño general en la tabla 4 , se observa que la modalidad normal presenta el $80 \%$ de los estudiantes en el nivel bajo. 


\begin{tabular}{|c|c|c|c|c|c|}
\hline \multirow{2}{*}{$\begin{array}{c}\text { Modalidad } \\
\text { Institución }\end{array}$} & \multicolumn{2}{|c|}{ Desempeño general } & \multicolumn{2}{c|}{ Puntaje Porcentual } \\
\cline { 2 - 6 } & Bajo & $\begin{array}{c}\text { Medio } \\
\text { bajo }\end{array}$ & Medio & Media & I. C. al 95\% \\
\hline Académico & $54,0 \%$ & $36,0 \%$ & $10,0 \%$ & 30,16 & $26,58-33,74$ \\
\hline Técnico & $30,0 \%$ & $60,0 \%$ & $10,0 \%$ & 34,20 & $28,80-39,60$ \\
\hline $\begin{array}{c}\text { Media } \\
\text { Técnica }\end{array}$ & $41,3 \%$ & $38,7 \%$ & $20,0 \%$ & 36,41 & $33,94-38,89$ \\
\hline Normal & $80,0 \%$ & $20,0 \%$ & $0,0 \%$ & 19,50 & $11,82-27,18$ \\
\hline
\end{tabular}

Tabla 4: Análisis por modalidad de la I. E.

Al analizar la actitud según la modalidad de la I. E. también se encontró que la modalidad normalista obtuvo el promedio de puntaje en actitud más bajo, sin embargo se encuentra en el nivel de actitud positiva al igual que las demás modalidades.

El análisis hecho a través del ANOVA de un factor para la prueba de conocimientos general $(p=0,001)$ y la prueba de actitud hacia la matemática $(p=0,006)$, muestran que existen diferencias significativas en las medias de las diferentes modalidades.

En la prueba de actitud hacia la matemática al realizar el contraste de medias se encontró que en todas las modalidades las medias no presentan diferencias significativas $(p=$ $0,070)$.

Análisis de la actitud hacia la matemática de los estudiantes:

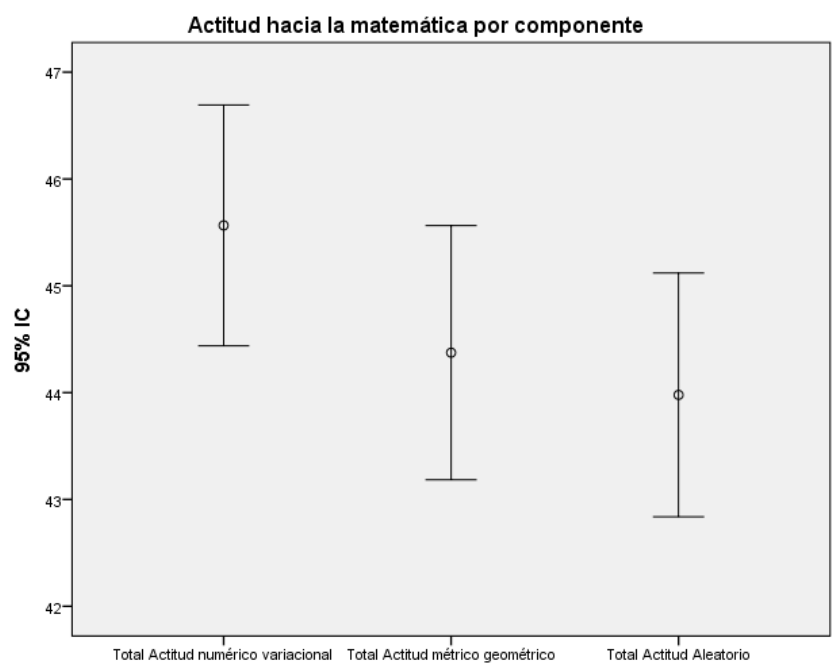

Ilustración 1: I. C de actitud matemática por componente

La valoración total del componente numérico - variacional fue la más alta de los 3 componentes. Sin embargo no se presentan diferencias significativas entre las medias de los 3 componentes, la valoración total de la actitud presenta un promedio de 133,70 puntos sobre 180 , la cual es favorable.

\section{Análisis de la actitud matemática por grados:}

Los resultados de la prueba de actitud hacia la matemática muestran que grado tercero presenta una actitud más favorable, la cual se encuentra en el rango de actitud muy positiva, mientras que los demás grados se encuentran en el nivel de actitud positiva, según la escala de actitud planteada para esta prueba.

Según la prueba chi-cuadrado de Pearson $(P<0.001)$, las variables grado y actitud matemática presentan una asociación significativa y según la correlación de Spearman $(\rho=-0.367$, con P- valor $<0,001)$, dicha asociación es inversa, es decir, a menor grado mejor nivel de actitud matemática.

Comprobados los supuestos de normalidad y homogeneidad de varianzas se realizó el análisis de varianza, encontrándose que existen diferencias significativas entre las medias de la actitud hacia la matemática, por lo cual se realizó la prueba de Scheffé para comparaciones múltiples, encontrándose que no existen diferencias significativas entre las medias de los grados $3^{\circ}$ y $5^{\circ}(p=0,392)$, ni entre las medias de los grados $9^{\circ}$ y $11^{\circ} \quad(p=0,999)$, entre los demás pares de comparaciones las diferencias son significativas.

\section{Análisis de la actitud matemática frente al desempeño general:}

Según la prueba chi-cuadrado de Pearson $(P=0.001)$, las variables actitud y desempeño general presentan una asociación significativa y según la correlación de Spearman ( $\rho$ $=0.290$, con P- valor $<0,001$ ), dicha asociación es directa, es decir, a mejor actitud el desempeño escolar es mejor.

Correlación entre la prueba de conocimientos generales en matemáticas y la prueba de actitud hacia la matemática:

Según la prueba de correlación de Pearson $(\rho=0,293)$ $(p<0,001)$ existe relación directa entre la actitud hacia la matemática y los resultados de la prueba en conocimientos matemáticos.

Análisis del puntaje porcentual promedio por pensamiento matemático en los profesores:

\begin{tabular}{|c|c|c|c|}
\hline \multirow{2}{*}{$\begin{array}{c}\text { Pensamiento } \\
\text { matemático }\end{array}$} & \multirow{2}{*}{ Media } & \multicolumn{2}{|c|}{$\begin{array}{c}\text { Intervalo de } \\
\text { confianza al 95\% }\end{array}$} \\
\cline { 3 - 4 } & $\begin{array}{c}\text { Límite } \\
\text { inferior }\end{array}$ & $\begin{array}{c}\text { Límite } \\
\text { superior }\end{array}$ \\
\hline Numérico & 70,11 & 64,00 & 76,22 \\
\hline Variacional & 80,00 & 73,19 & 86,81 \\
\hline Métrico & 74,67 & 67,89 & 81,44 \\
\hline Geométrico & 74,84 & 67,76 & 81,92 \\
\hline Aleatorio & 68,84 & 60,42 & 77,26 \\
\hline Total & 73,37 & 68,96 & 77,77 \\
\hline
\end{tabular}


Tabla 5: Puntaje por pensamiento en profesores

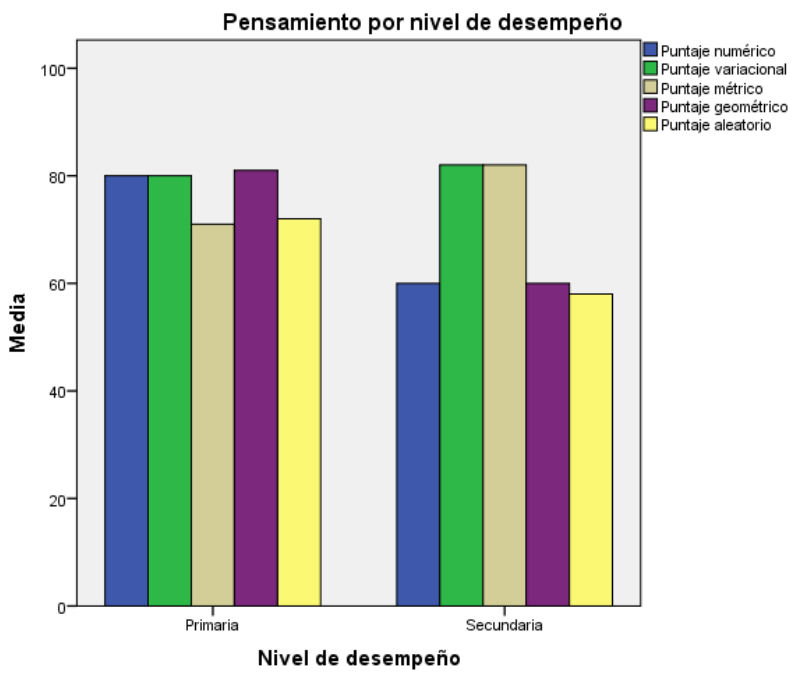

Ilustración 2: Nivel de desempeño por pensamiento

El puntaje promedio en conocimientos matemáticos en el total general de la prueba para los profesores de primaria se encuentra en $72,71 \%$ y para los profesores de secundaria en $74,51 \%$, lo cual corresponde a un desempeño alto según la clasificación del ICFES, pero equivale a un desempeño bajo según las hipótesis planteadas para esta investigación ya que se evaluó sobre los conceptos básicos en estos niveles y las pruebas aplicadas a los docentes fueron las mismas de los estudiantes de grado quinto y grado once como se explicó anteriormente.

Análisis del puntaje de la prueba general y de la prueba de actitud matemática frente al sexo:

Se realizó una prueba $T$ para muestras independientes y según el estadístico de Levene, existe homogeneidad de varianzas en los totales de la prueba de conocimientos matemáticos $(p=$ $0,374)$, y en la prueba de actitud matemática $(p=0,284)$, y según la prueba $\mathrm{T}$ no existen diferencias significativas entre las medias en el total de la prueba de conocimientos matemáticos $(p=0,804)$, pero si existen diferencias significativas entre las medias en la prueba de actitud matemática $(p=0,047)$ entre hombres y mujeres, siendo mejor la actitud de las mujeres que la de los hombres.

\section{Análisis del promedio en conocimientos y actitud matemática según el nivel de estudios:}

Según el estadístico de Levene, existe homogeneidad de varianzas en el total de la prueba de conocimientos matemáticos $(p=0,293)$ y en la prueba de actitud matemática $(p=0,123)$, y según la prueba $\mathrm{T}$ no existen diferencias significativas entre las medias del total de la prueba de conocimientos matemáticos $(p=0,107)$ según la formación profesional, pero si existen diferencias significativas en las medias de la prueba de actitud matemática $(p=0,037)$, según la formación profesional o con post grado de los profesores de primaria y secundaria.

\section{Análisis del promedio en conocimientos y actitud} matemática según la profesión:

Según la prueba de Levene, existe homogeneidad de varianzas en el total de la prueba de conocimientos matemáticos ( $p$ $=0,438)$ y en la prueba de actitud matemática $(p=0,319)$, de acuerdo a la profesión y según la prueba $\mathrm{T}$ no existen diferencias significativas entre las medias del total de la prueba de conocimientos matemáticos $(p=0,311)$ y la prueba de actitud matemática $(p=0,290)$, de acuerdo a la profesión de los profesores. Esto teniendo en cuenta que la mayoría de los profesores encuestados son de profesión licenciados.

\section{Correlación entre variables:}

En todos los casos $p$-valor $>=0,05$ lo que indica que no existe relación entre la variable edad y el puntaje de la prueba de conocimientos matemáticos, ni entre la edad y la actitud matemática.

Tampoco existe relación entre el tiempo de experiencia en la docencia con respecto al total de la prueba de conocimientos matemáticos y a la prueba de actitud matemática.

\section{Prueba de actitud matemática en profesores:}

$\mathrm{Al}$ analizar los puntajes obtenidos en cada uno de los ítems, se puede observar que la valoración de la actitud en todos los componentes matemáticos es superior a 4 , excepto en dos ítems relacionados con geometría con puntajes de 3,88 y 3,98.

$\mathrm{Al}$ analizar la valoración de la actitud en los estratos primaria y secundaria, se encontró que en todos los componentes matemáticos y en la prueba general, la actitud obtuvo valoración alta.

Al analizar los niveles de actitud matemática de los docentes, se encontró que la mayoría presentan una actitud matemática muy positiva, especialmente los docentes de secundaria.

Sólo el 4,5\% de los docentes encuestados presentan una actitud negativa hacia la matemática y solo el 2,2\% presenta una actitud indiferente.

\section{Análisis multivariado profesores:}

El análisis de correspondencias múltiples explican el 71,55\% de la inercia total, con los primeros tres ejes factoriales.

En este análisis se observa que el primer plano factorial (eje 1 Vs. Eje 2) y en el segundo plano factorial (eje 1 Vs. Eje 3) presentan características semejantes, el primer eje está definido por el sexo y el nivel de desempeño, en el lado izquierdo se observa los docentes de primaria mujeres de 
profesión licenciadas, con actitud positiva hacia la matemática y desempeño de nivel medio, en el lado derecho se observan los docentes de secundaria, en su mayoría hombres con post grado, con actitud muy positiva hacia la matemática y desempeño alto; en su gran mayoría los docentes son de profesión licenciados, son muy pocos los docentes de otras profesiones que trabajan en la docencia.

En el tercer plano factorial (eje 2 Vs.eje 3) el primer eje se define por el nivel profesional, en el lado izquierdo se observa las mujeres que se desempeñan en secundaria, licenciadas con post grado con una actitud muy positiva hacia la matemática y con desempeño alto, en el lado derecho se encuentran los hombres con actitud positiva, profesionales de otras ramas, con desempeño medio.

\section{CONCLUSIONES}

1. Los errores conceptuales de ejemplificación y definición detectados en los estudiantes en los niveles inferiores como $3^{\circ}$ y $5^{\circ}$, permanecen hasta los estudiantes de grado once en los niveles intelectuales de interpretación y argumentación.

2. El nivel de conocimientos matemáticos de los estudiantes, de acuerdo a la prueba empleada es bajo, con aproximadamente el $87 \%$ de estudiantes ubicados en el nivel bajo y medio bajo, lo que corresponde a puntajes por debajo del $50 \%$.

3. El pensamiento numérico es el que presenta puntajes más bajos, su media corresponde al nivel insuficiente según la clasificación del Icfes, a pesar de ser uno de los pensamientos al que más horas de clase se le dedica.

4. Menos del $1 \%(0,87 \%)$ de los estudiantes encuestados presentan un desempeño en nivel alto.

5. La actitud hacia la matemática influye directamente y en un nivel medio en los resultados de la prueba de conocimientos, es decir, a mejor actitud mejores resultados en la prueba de conocimientos matemáticos, sin embargo se puede pensar que el aprendizaje además de verse afectado por la actitud, tiene asociación con otras variables.

6. En general los estudiantes presentan una actitud positiva hacia la matemática en todos los grados, no se presentaron actitudes muy negativas y sólo el 1,3\%, de la población encuestada presentó actitud negativa hacia la matemática.

7. Las variables grado y actitud hacia la matemática, presentan una asociación inversa, la fuerza de esta asociación es media, esta misma asociación se presenta entre la actitud y la variable edad, pues estas son directamente proporcionales, es decir, al aumentar el grado también aumenta la edad.

8. No existe relación entre el sexo y los resultados de las pruebas, esto puede deberse a que ambos sexos recién el mismos tipo de educación.

9. No hay diferencias significativas entre las medias de los grados $3^{\circ}$ y $5^{\circ}$, ni entre las medias de $9^{\circ}$ y $11^{\circ}$, pero si existe diferencia significativa entre las medias de primaria y secundaria con respecto al puntaje general.

10. La modalidad influye en los resultados de la prueba de conocimientos matemáticos, siendo la media técnica la de mejor puntaje promedio $(36,41)$, lo cual equivale a un desempeño medio bajo, y la normalista la de puntaje promedio más bajo $(19,50)$ que equivale a un desempeño bajo, en especial en los pensamientos métrico, geométrico y aleatorio, esto se podría deber a que las instituciones educativas disminuyen la intensidad horaria de áreas básicas como la matemática, para dar horas a asignaturas de la modalidad, por lo general esta disminución de horas se refleja en que los temas de medida, geometría y estadística se dictan en muy corto tiempo o no se alcanzan a dictar en el año respectivo.

11. La IE con modalidad normalista presenta los resultados más bajos en las pruebas de conocimiento con un $80 \%$ de la población estudiantil en nivel bajo y $20 \%$ en nivel medio bajo, lo cual es contradictorio debido a que estos estudiantes serán fututos docentes, pero esto podría deberse al énfasis pedagógico de la institución.

12. Hay diferencias significativas entre las medias de la prueba de conocimientos matemáticos en las diferentes modalidades de la institución, pero no hay diferencias significativas entre las medias de la actitud hacia la matemática.

13. Algunos de los errores conceptuales encontrados en los estudiantes, se presentan en:

a. El pensamiento numérico, en operaciones con conjuntos, identificación de posiciones decimales, fracciones

b. El pensamiento variacional, en secuencias lógicas, proporcionalidad, planteamiento de ecuaciones, concepto de función y límites.

c. El pensamiento métrico, en perímetro, áreas, volúmenes, conversión de unidades y ley de seno y coseno.

d. El pensamiento geométrico, en clasificación de ángulos y triángulos, identificación de figuras geométricas y cuerpos geométricos, rotación de figuras, semejanza y congruencia, teorema de Pitágoras. 
e. El pensamiento aleatorio, en interpretación de gráficos y tablas de frecuencias, técnicas de conteo, medidas de tendencia central y probabilidad.

14. Los resultados de las pruebas practicadas a los docentes de primaria y del área de matemáticas, muestran un desempeño de nivel bajo (inferior al $80 \%$ ), teniendo en cuenta que los docentes de primaria fueron encuestados con los mismos instrumentos aplicados a los estudiantes de grado quinto y los docentes de secundaria con los instrumentos de grado once, es decir la finalidad de la prueba era determinar el nivel de conocimientos básicos que poseen los docentes para orientar tanto primaria como secundaria.

15. La actitud hacia la matemática que presentan los docentes de primaria y secundaria es muy favorable, siendo más favorable la actitud de los docentes de secundaria

16. Algunos de los errores conceptuales encontrados en los estudiantes, que también se perciben en una mínima parte de la población docente, son:

a. En pensamiento numérico, en operaciones con conjuntos e identificación de posiciones decimales.

b. En pensamiento variacional, en secuencias lógicas y proporcionalidad.

c. En pensamiento métrico, en áreas y conversión de unidades.

d. En pensamiento geométrico, clasificación de ángulos y triángulos.

e. En pensamiento aleatorio, en interpretación de gráficos y tablas de frecuencias, técnicas de conteo, medidas de tendencia central y probabilidad.

\section{RECOMENDACIONES}

1. Es necesario y urgente un cambio de fondo en las estructuras educativas, pareciera que con el transcurrir del tiempo, a pesar de los múltiples avances de la ciencia y la tecnología, la educación fuera en un retroceso. Se habla de aprendizaje por competencias, pero la verdad es que nuestros estudiantes están saliendo del ciclo de educación media sin las competencias mínimas necesarias para enfrentar los retos actuales y competir por su lugar en una sociedad cada vez más demandante, pero que pocas oportunidades les brinda.

2. Sería muy conveniente que desde la educación primaria se pudiese trabajar por áreas, y ojalá por docentes expertos en cada área como sucede en los países más desarrollados en educación como Japón, Finlandia, entre otros, esto evitaría la transmisión de errores conceptuales desde temprana edad.
3. Se deben implementar estrategias de aprendizaje con los estudiantes y renovar las metodologías de enseñanza, los jóvenes viven una era tecnológica y por lo tanto es necesario cambiar los métodos tradicionales por clases donde se utilicen las nuevas tecnologías y los recursos tecnológicos actuales, que capten el interés de los estudiantes, pero para ello las instituciones educativas oficiales deben contar con los recursos suficientes para satisfacer estas necesidades.

4. Los planes y estrategias que el gobierno nacional propone en cuanto a cobertura y calidad son buenas, pero la realidad en las aulas es otra, los factores sociales, económicos y culturales con que conviven los jóvenes, son el principal obstáculo que los docentes libran cada día. Mientras nuestros estudiantes no sean motivados por aspiraciones futuras mejores, será muy difícil alcanzar las metas de calidad educativa anheladas.

\section{AGRADECIMIENTOS}

Agradecimientos especiales al Profesor Mg. Robín Mario Escobar y al Semillero de Investigación en Educación Matemática.

\section{REFERENCIAS}

[1]. ICFES. Estudio Internacional de Tendencias en Matemática y Ciencias. Resultados de TIMSS 2007, Resumén ejecutivo de los Resultados de Colombia. [En línea] 06 de 2014. www.icfes.gov.co.

[2]. —. Resultados Nacionales en Pruebas PISA. [En línea] 06 de 2014. http//www.icfes.gov.co/investigación/evaluacionesinternacionales/pisa..

[3]. Acevedo, Myriam, y otros. [aut. libro] Instituto Colombiano para el Fomento de la Educación SuperiorICFES. Fundamentación Conceptual Área de Matemáticas. Bogotá : s.n., 2007.

[4]. Ministerio de Educación Nacional, Colombia. Resultados Nacionales 2009 - 2012. Pruebas Saber Matemáticas. [En línea] 06 de 2014. www.icfes.gov.co. 